\title{
Isospectrality of spherical MHD dynamo operators: Pseudo-Hermiticity and a no-go theorem
}

\author{
Uwe Günther* and Frank Stefani ${ }^{\dagger}$ \\ Research Center Rossendorf, P.O. Box 510119, D-01314 Dresden, Germany
}

7 August 2002

\begin{abstract}
The isospectrality problem is studied for the operator of the spherical hydromagnetic $\alpha^{2}-$ dynamo. It is shown that this operator is formally pseudo-Hermitian ( $J$-symmetric) and lives in a Krein space. Based on the $J$-symmetry, an operator intertwining Ansatz with first-order differential intertwining operators is tested for its compatibility with the structure of the $\alpha^{2}$-dynamo operator matrix. An intrinsic structural inconsistency is obtained in the set of associated matrix Riccati equations. This inconsistency is interpreted as a no-go theorem which forbids the construction of isospectral $\alpha^{2}$-dynamo operator classes with the help of first-order differential intertwining operators.
\end{abstract}

\section{Introduction}

The magnetic fields of stars and planets are generated by the homogeneous dynamo effect in moving electrically conducting fluids [1]. This effect is explained within the framework of magneto-hydrodynamics (MHD), but its experimental demonstration was missing until recently. In 1999, the first successful dynamo experiments in Riga and Karlsruhe [2] opened up a new way for the laboratory investigation of homogeneous dynamos. In connection with the data analysis for the existing experiments and the design of new dynamo experiments there is a growing interest in the spectral properties of dynamos. Of particular interest is the question whether isospectral dynamos can exist. First numerical results on this topic were published in 3 , 4, but rigorous results are still missing.

As a step towards clarification of this issue, we study in the present paper the question whether operator intertwining techniques from quantum mechanics (QM) can be adopted to MHD dynamo models. In case of an affirmative answer we would obtain an efficient tool for constructing isospectral classes of MHD dynamo operators. Otherwise we would get a no-go theorem which would forbid a straight analogy with quantum mechanical models.

Let us start by recalling some essentials of operator intertwining transformations in QM [5]. Two operators $H_{0}$ and $H_{1}$ are said to be intertwined if there exist operators $A_{+}$and $A_{-}$so that

$$
H_{1} A_{+}=A_{+} H_{0}, \quad A_{-} H_{1}=H_{0} A_{-} .
$$

For the corresponding eigenfunctions $\phi_{0}$ and $\phi_{1}$ holds, up to normalization,

$$
\phi_{1}=A_{+} \phi_{0}, \quad A_{-} \phi_{1}=\phi_{0}
$$

and the operators $H_{0}$ and $H_{1}$ are isospectral, except for those states that are annihilated by $A_{+}$or $A_{-}$. In the case of one-dimensional Schrödinger operators $H_{0}=p^{2}+V_{0}(x)$ and $H_{1}=p^{2}+V_{1}(x)$ with the momentum operator given as $p=-i \partial_{x}$, the intertwining operators can be chosen as first order differential operators

$$
A_{+}=A:=i p+f, \quad A_{-}=A^{\dagger}=-i p+f .
$$

Structural compatibility of $H_{0}$ and $H_{1}$ with the intertwining relations (1) requires that the function $f(x)$ and the potentials $V_{0}(x), V_{1}(x)$ are connected by the consistency conditions

$$
\begin{aligned}
V_{1} & =V_{0}+2 f^{\prime} \\
-f^{\prime}+f^{2} & =V_{0}-E \\
f^{\prime}+f^{2} & =V_{1}-E,
\end{aligned}
$$

*e-mail: u.guenther@fz-rossendorf.de

$\dagger$ e-mail: f.stefani@fz-rossendorf.de 
where the prime denotes differentiation with respect to $x$; and $E$ is a constant of integration. Linearization of the Riccati differential equations (3), (4) shows that this constant can be interpreted as eigenvalue of the Schrödinger operators $H_{0}$ and $H_{1}$

$$
\begin{array}{ll}
H_{0} \chi_{0}=E \chi_{0} & \text { for } \quad f=-\frac{\chi_{0}^{\prime}}{\chi_{0}}, \\
H_{1} \chi_{1}=E \chi_{1} & \text { for } \quad f=\frac{\chi_{1}^{\prime}}{\chi_{1}},
\end{array}
$$

where $\chi_{0}$ and $\chi_{1}$ are formal, and not necessarily normalized eigenfunctions of $H_{0}$ and $H_{1}$, respectively. They are connected by the product relation

$$
\chi_{0} \chi_{1}=c
$$

with $c$ a non-vanishing constant. It is straightforward to verify that the shifted Schrödinger operators are factorizable in terms of the intertwining operators

$$
H_{0}-E=A^{\dagger} A, \quad H_{1}-E=A A^{\dagger} .
$$

First-order differential intertwining transformations of type (2) are known as Darboux transformations [ 6 and are widely used to generate isospectral operator classes from given operators with known spectra [5, 17, 8]. In particular, intertwining constructions are a basic ingredient of super-symmetric quantum mechanical models [5, 9] and their generalizations to pseudo-supersymmetric systems [10, 11]. As it was demonstrated in [12, a double-intertwining (double commutation) method can provide a tool for inserting additional eigenvalues in spectral gaps of given background Schrödinger and Jacobi operators.

Motivated by the large number of exact results on isospectral classes obtained by operator intertwining constructions, it is natural to investigate whether MHD dynamo operators are also suitable for this technique. For this purpose we study in the present paper the simplest mean-field MHD dynamo configuration - the spherical $\alpha^{2}$-dynamo [1]. In terms of the radial momentum operator $p=-i\left(\partial_{r}+1 / r\right)$ the $2 \times 2$ operator matrix of the $\alpha^{2}$-dynamo is given as $^{1}$

$$
\hat{H}_{l}[\alpha] \equiv\left(\begin{array}{rr}
-p^{2}-\frac{l(l+1)}{r^{2}} & \alpha(r) \\
p \alpha(r) p+\alpha(r) \frac{l(l+1)}{r^{2}} & -p^{2}-\frac{l(l+1)}{r^{2}}
\end{array}\right)
$$

and lives on the domain

$$
\begin{gathered}
\mathcal{D}\left(\hat{H}_{l}[\alpha]\right):=\left\{\psi=\left(\begin{array}{l}
\psi_{1} \\
\psi_{2}
\end{array}\right): \psi \in \tilde{\mathcal{H}} \equiv \mathcal{H} \oplus \mathcal{H}, \mathcal{H}=L_{2}\left(\Omega, r^{2} d r\right),\right. \\
\left.\Omega=[0,1], \psi(1)=0,\left.r \psi(r)\right|_{r \rightarrow 0} \rightarrow 0\right\}
\end{gathered}
$$

in the Hilbert space $\tilde{\mathcal{H}}$. It describes the coupled $l$-modes of the poloidal and toroidal magnetic field components in a mean-field dynamo model with helical turbulence function $\alpha(r)$. The function $\alpha(r)$ does not depend on $l$ and we assume that it is real-valued, positive definite, bounded, and sufficiently smooth on $\Omega$ : $\operatorname{Im} \alpha=0,0<$ $\alpha \leq c_{1}<\infty, \alpha \in C^{4}(\Omega)$. The idealized boundary condition $\psi(r=1)=0$ corresponds to a super-conducting spherical boundary shell and is chosen here to ensure simplicity of the subsequent considerations [13. For more realistic models with close relation to stellar dynamos, the spherical fluid configurations confined to $r<1$ can be assumed as embedded in empty space. The boundary condition should then be replaced by $\left.\hat{B}_{l} \psi\right|_{r=1}=0$ with $\hat{B}_{l}=\operatorname{diag}\left[\partial_{r}+(l+1) / r, 1\right]$ (see e.g. [1]) what will require a more general approach than that presented in the present paper.

Exploring the fundamental symmetry of the $\alpha^{2}$-dynamo operator matrix we find in section 2 that $\hat{H}_{l}[\alpha]$ acts as a symmetric operator on the Hilbert space $\tilde{\mathcal{H}}$ when this is endowed with an indefinite metric $J$. I.e., the $\alpha^{2}$-dynamo operator matrix is a $J$-symmetric (formally $J$-self-adjoint) operator

$$
\hat{H}_{l}[\alpha]=\hat{H}_{l}^{\sharp}[\alpha]:=J \hat{H}_{l}^{\dagger}[\alpha] J
$$

living in a Krein space $\tilde{\mathcal{K}}=\tilde{\mathcal{H}}_{J}$ 14. $J$-self-adjointness is a natural property of operators from different fields of physics. Examples are, e.g., the super-symmetric Dirac operator [15], PT-symmetric non-Hermitian

\footnotetext{
${ }^{1} \mathrm{~A}$ brief outline of the derivation of the $\alpha^{2}$-dynamo operator matrix from the MHD mean-field induction equation can be found in Appendix $\mathrm{A}$
} 
Hamiltonians in QM [10, 16] as well as the Wheeler-DeWitt operator for a cosmological Friedman-RobertsonWalker model coupled to a real massive scalar field [10. Since the recent paper series [10, 11, 17] of A. Mostafazadeh on non-Hermitian operators with real spectra, $J$-self-adjoint operators are also known as pseudoHermitian operators.

In analogy with the simple quantum mechanical model described above, we base our isospectrality analysis on an intertwining Ansatz for two $\alpha^{2}$-dynamo operators with helical turbulence functions $\alpha_{0}(r), \alpha_{1}(r)$

$$
\hat{H}_{l_{0}}\left[\alpha_{0}\right]-E I=-\hat{A} \hat{A}^{\sharp}, \quad \hat{H}_{l_{1}}\left[\alpha_{1}\right]-E I=-\hat{A}^{\sharp} \hat{A}
$$

and intertwining operator matrices $\hat{A}, \hat{A}^{\sharp}$ that are first-order differential operators

$$
\hat{A}:=i R(r) p+Q(r), \quad \hat{A}^{\sharp}:=-i p R^{\sharp}(r)+Q^{\sharp}(r) .
$$

This Ansatz leads to a set of six consistency conditions on the matrices $R(r)$ and $Q(r)$ which are studied in section 3. It is shown that one pair of conditions fixes the structure of $R(r)$ in terms of the helical turbulence functions $\alpha_{0}(r)$ and $\alpha_{1}(r)$. A second pair is equivalent to the symmetry relations $B=B^{\sharp}, U=U^{\sharp}$ on the matrix functions

$$
\begin{aligned}
& B:=R^{\sharp} Q \\
& U:=R\left[Q^{\sharp}-\left(R^{\sharp}\right)^{\prime}\right]=R B R^{-1}-R\left(R^{\sharp}\right)^{\prime}
\end{aligned}
$$

and can be regarded as an implicit consequence of the $J$-pseudo-Hermiticity of the operator matrices $\hat{H}_{l_{0}}\left[\alpha_{0}\right]$ and $\hat{H}_{l_{1}}\left[\alpha_{1}\right]$. (The prime denotes the derivative with respect to $r$.) The remaining two conditions can be transformed into a pair of coupled matrix Riccati differential equations (MREs) on $B$ and $U$.

The consistency of the six conditions is analyzed in section 4 with the help of a step-by-step reduction of their complexity. First, we conclude from the limiting behavior of the MREs for $r \rightarrow 0$ that the angular mode numbers $l_{0}$ and $l_{1}$ in the two dynamo operator matrices should be connected by the incremental relation $l_{1}=l_{0}+1$. Then we use the $J$-symmetry of $B$ to derive from the coupled MREs a system of coupled non-linear ordinary differential equations (ODEs) involving the helical turbulence functions $\alpha_{0}(r)$ and $\alpha_{1}(r)$. Analyzing these ODEs we are able to show the existence of an inherent contradiction between them. As implication, we arrive at a no-go theorem which states that the six consistency conditions cannot be fulfilled simultaneously and that, hence, the structure of the $\alpha^{2}$-dynamo operator matrices is not suitable for an operator intertwining technique based on an Ansatz with first-order differential intertwining operators.

In the concluding section 5 we briefly discuss some other methods which could be useful for studying isospectrality issues of the dynamo operator matrix and which possibly could provide a technique to construct classes of isospectral spherical $\alpha^{2}$-dynamo operators.

\section{$2 \quad J$-Symmetry of the dynamo operator matrix}

In this section, we study the fundamental symmetry $J$ of the $\alpha^{2}$-dynamo operator matrix (8) what allows us to choose an appropriate Ansatz for the intertwining operators $A_{+}$and $A_{-}$.

We start our consideration by introducing the auxiliary operator

$$
Q[\alpha]:=p \alpha p+\alpha \frac{l(l+1)}{r^{2}}
$$

defined on the domain

$$
\mathcal{D}(Q)=\left\{\phi: \phi \in \mathcal{H}=L_{2}\left(\Omega, r^{2} d r\right), \phi(1)=0,\left.\quad r \phi(r)\right|_{r \rightarrow 0} \rightarrow 0\right\}
$$

in the Hilbert space $\mathcal{H}$. The operator $Q[\alpha]$ is a formally self-adjoint ${ }^{2}$ singular differential operator $Q=Q^{\dagger}$ which acts as symmetric operator on $\mathcal{H}$. In terms of $Q[\alpha]$ the dynamo operator matrix and its formal adjoint read

$$
\hat{H}_{l}[\alpha]=\left(\begin{array}{cc}
-Q[1] & \alpha \\
Q[\alpha] & -Q[1]
\end{array}\right), \quad \hat{H}_{l}^{\dagger}[\alpha]=\left(\begin{array}{cc}
-Q[1] & Q[\alpha] \\
\alpha & -Q[1]
\end{array}\right)
$$

\footnotetext{
${ }^{2}$ In the subsequent compatibility analysis of the operator intertwining construction we restrict our attention to symmetric (formally self-adjoint) operators. For simplicity, we leave questions of self-adjoint extensions and corresponding generalized boundary conditions 18 19 for the bi-component functions $\psi$ aside.
} 
so that the fundamental (canonical) symmetry can be obtained as

$$
\hat{H}_{l}[\alpha]=\hat{H}_{l}^{\sharp}[\alpha]:=J \hat{H}_{l}^{\dagger}[\alpha] J, \quad J=\left(\begin{array}{cc}
0 & 1 \\
1 & 0
\end{array}\right) .
$$

Diagonalizing the matrix $J$

$$
J \longrightarrow \eta=S^{T} J S, \quad S=\frac{1}{\sqrt{2}}\left(\begin{array}{rr}
1 & -1 \\
1 & 1
\end{array}\right), \quad \eta=\left(\begin{array}{rr}
1 & 0 \\
0 & -1
\end{array}\right)
$$

we see that $\hat{H}_{l}[\alpha]$ is equivalent to the operator matrix

$$
\check{H}_{l}[\alpha]=S^{T} \hat{H}_{l}[\alpha] S=\frac{1}{2}\left(\begin{array}{cc}
Q[\alpha-2]+\alpha & -Q[\alpha]+\alpha \\
Q[\alpha]-\alpha & Q[-\alpha-2]-\alpha
\end{array}\right)
$$

with the property $\check{H}_{l}[\alpha]=\eta \check{H}_{l}[\alpha]^{\dagger} \eta$. The fundamental $\eta$-symmetry of the operator matrix $\check{H}_{l}[\alpha]$ implies that $\mathcal{D}\left(\check{H}_{l}\right)$ could be endowed with the indefinite metric $\eta$ so that $\check{H}_{l}[\alpha]$ becomes a symmetric operator on $\mathcal{D}\left(\check{H}_{l}\right)$. Due to the invariance of the signature under the transformation $S$ the domain $\mathcal{D}\left(\hat{H}_{l}\right)$ can also be endowed with a natural indefinite inner product $[\cdot, \cdot]_{J}$ defined by the metric $J$

$$
[x, y]_{J}:=(x, J y), \quad x, y \in \tilde{\mathcal{H}}=\mathcal{H} \oplus \mathcal{H},
$$

where $(\cdot, \cdot)$ denotes the usual inner (scalar) product in the Hilbert space $\tilde{\mathcal{H}}$. This means that $\hat{H}_{l}[\alpha]$ is a $J$-symmetric operator which acts as symmetric operator in a Krein space $^{3} \tilde{\mathcal{K}}=\tilde{\mathcal{H}}_{J}$

$$
\left[\hat{H}_{l} x, y\right]_{J}=\left[x, \hat{H}_{l}^{\sharp} y\right]_{J} .
$$

From its operator-matrix representation (9) we see that $J$ is self-adjoint, involutory and unitary

$$
J^{\dagger}=J, \quad J^{2}=I, \quad J^{-1}=J^{\dagger}
$$

so that $\hat{H}_{l}[\alpha]$ is a $J$-pseudo-Hermitian operator in the sense of [10, 11, 17.

The eigenvalues of $J$-pseudo-Hermitian operators are known [10, 14, to be either real or to come in complex-conjugate pairs. Here we illustrate this property by passing from the eigenvalue problem for the linear operator pencil

$$
\hat{L}_{l}[\alpha, \lambda] \psi:=\left(\hat{H}_{l}[\alpha]-\lambda\right) \psi=0
$$

to the eigenvalue problem $L_{l}[\alpha, \lambda] \psi_{1}=0$ for the associated quadratic operator pencil $L_{l}[\alpha, \lambda]$. This pencil can be derived explicitly from the Ansatz

$$
\psi=\left(\begin{array}{r}
\psi_{1} \\
\frac{1}{\alpha}[Q(1)+\lambda] \psi_{1}
\end{array}\right)
$$

with $\alpha(r) \neq 0$. As result we obtain

$$
\begin{aligned}
L_{l}[\alpha, \lambda] \psi_{1} & \equiv\left\{[Q[1]+\lambda] \frac{1}{\alpha}[Q[1]+\lambda]-Q[\alpha]\right\} \psi_{1}=0 \\
& =\left(A_{2} \lambda^{2}+A_{1} \lambda+A_{0}\right) \psi_{1}=0 .
\end{aligned}
$$

The operators

$$
A_{0}:=Q[1] \frac{1}{\alpha} Q[1]-Q[\alpha], \quad A_{1}:=Q[1] \frac{1}{\alpha}+\frac{1}{\alpha} Q[1], \quad A_{2}:=\frac{1}{\alpha}
$$

are formally self-adjoint on $\mathcal{D}(Q)$ so that the functionals $a_{j}:=\left(A_{j} \psi_{1}, \psi_{1}\right), j=1,2,3$ are real-valued: $\operatorname{Im} a_{j}=0$.

From the quadratic equation

$$
\left(L_{l}[\alpha, \lambda] \psi_{1}, \psi_{1}\right)=a_{2} \lambda^{2}+a_{1} \lambda+a_{0}=0
$$

\footnotetext{
${ }^{3}$ For surveys on operators in Krein spaces (Hilbert spaces with additional indefinite inner product structures) we refer to the mathematical literature 14 20].
} 
we conclude that the eigenvalues of the $J$-pseudo-Hermitian dynamo operator matrix $\hat{H}_{l}$ and its associated pencil $L_{l}$ occur as eigenvalue pairs [21]

$$
\lambda_{ \pm}=\frac{1}{2 a_{2}}\left(-a_{1} \pm \sqrt{a_{1}^{2}-4 a_{0} a_{2}}\right) .
$$

Obviously, the sign of the discriminant $\Delta:=a_{1}^{2}-4 a_{0} a_{2}$ defines whether $\lambda_{ \pm}$are both real or pairwise complex conjugate. The transition from real eigenvalues $\lambda_{ \pm}$to complex ones occurs at $\Delta=0$ where the eigenvalue becomes two-fold degenerate $\lambda_{+}=\lambda_{-}=\lambda_{0}=-\frac{a_{1}}{2 a_{2}}$. This general behavior of $\lambda_{ \pm}$confirms the results of numerical simulations [4, which showed that a scaling of the helical turbulence function $\alpha$ leads to a pairwise intersection of real eigenvalue branches of $\hat{H}_{l}$ and a transition at the intersection points to a pair of complex conjugate eigenvalues.

We note that at the two-fold degenerate points $\lambda_{0}$ of the spectrum with $\Delta=0$ a Jordan-Keldysh chain 22 ] exists for the linear operator pencil

$$
\hat{L}_{l}\left(\lambda_{0}\right) \psi=0, \quad \hat{L}_{l}\left(\lambda_{0}\right) \chi=\psi
$$

as well as for the quadratic operator pencil

$$
L_{l}\left(\lambda_{0}\right) \psi_{1}=0, \quad L_{l}\left(\lambda_{0}\right) \chi_{1}+\partial_{\lambda} L_{l}\left(\lambda_{0}\right) \psi_{1}=0 .
$$

Both are built up from eigenvectors $\psi, \psi_{1}$ and associated vectors $\chi, \chi_{1}$, respectively.

\section{Consistency conditions and matrix Riccati equations}

The fundamental $J$-symmetry ( $J$-pseudo-Hermiticity) of the $\alpha^{2}$-dynamo operator matrix provides a natural Ansatz for an intertwining construction which respects this symmetry:

$$
\hat{H}_{l_{0}}\left[\alpha_{0}\right]-E I=-\hat{A} \hat{A}^{\sharp}, \quad \hat{H}_{l_{1}}\left[\alpha_{1}\right]-E I=-\hat{A}^{\sharp} \hat{A} .
$$

In general, the operator matrix $\hat{A}$ could be an $n$ th-order differential operator of the form

$$
\hat{A}=\sum_{k=1}^{n} R_{k}(r)(i p)^{k}+Q(r)
$$

with $2 \times 2$ matrices $R_{k}(r)$ and $Q(r)$ as coefficients. For simplicity, we restrict our attention in the present paper to the first-order differential operator

$$
\hat{A}=i R(r) p+Q(r)
$$

with $J$-adjoint $A^{\sharp}=-i p R^{\sharp}(r)+Q^{\sharp}(r)$. Here we define the $\sharp$-operation for a given $2 \times 2$ matrix $C$ as

$$
C^{\sharp}=J C^{\dagger} J=J C^{* T} J .
$$

Asterisk and superscript " $T "$ denote complex conjugation and transposition, respectively.

Let us introduce the abbreviations

$$
\begin{aligned}
K_{0,1} & :=I-\alpha_{0,1} \sigma_{-} \\
M_{0,1} & :=K_{0,1} \frac{l_{0,1}\left(l_{0,1}+1\right)}{r^{2}}+E I-\alpha_{0,1} \sigma_{+}
\end{aligned}
$$

with the nilpotent matrices $\sigma_{ \pm}$defined as $\sigma_{+}:=\left(\begin{array}{ll}0 & 1 \\ 0 & 0\end{array}\right), \quad \sigma_{-}:=\left(\begin{array}{ll}0 & 0 \\ 1 & 0\end{array}\right)$. The shifted $\alpha^{2}-$ dynamo operator matrices in (10) take then the short form

$$
\hat{H}_{l_{0,1}}\left[\alpha_{0,1}\right]-E I=-p K_{0,1} p-M_{0,1} .
$$

Substituting (11) and (12) into the intertwining Ansatz (10), making use of commutation relations like $[p, R(r)]=$ $-i R^{\prime}(r)$ and equating the coefficient matrices of the $p^{2}, p, I$ terms we obtain the following six consistency 
conditions

$$
\begin{aligned}
& \hat{H}_{l_{0}}: \quad p^{2}: \\
& R R^{\sharp}=K_{0} \\
& p: \quad R Q^{\sharp}-Q R^{\sharp}-R\left(R^{\sharp}\right)^{\prime}+R^{\prime} R^{\sharp}=0 \\
& I: \quad Q Q^{\sharp}-R\left(R^{\sharp}\right)^{\prime \prime}+R\left(Q^{\sharp}\right)^{\prime}-Q\left(R^{\sharp}\right)^{\prime}=M_{0} \\
& \hat{H}_{l_{1}}: \quad p^{2}: \quad \quad R^{\sharp} R=K_{1} \\
& p \text { : } \\
& -R^{\sharp} Q+Q^{\sharp} R=0 \\
& I: \quad Q^{\sharp} Q-\left(R^{\sharp} Q\right)^{\prime}=M_{1} \text {. }
\end{aligned}
$$

For a successful intertwining construction these matrix equations should be fulfilled simultaneously. So, the main task consists in finding explicit solution sets for (13) - (18). Alternatively, we should obtain intrinsic contradictions within this equation system which could be interpreted as a no-go theorem forbidding this construction for $\alpha^{2}$-dynamo operator matrices.

We start our analysis with eqs. (13) and (16). From the tautologies $R R^{\sharp} R=R R^{\sharp} R$ and $R^{\sharp} R R^{\sharp}=R^{\sharp} R R^{\sharp}$ follows

$$
R K_{1}=K_{0} R, \quad K_{1} R^{\sharp}=R^{\sharp} K_{0}
$$

what with

$$
R=\left(\begin{array}{ll}
r_{11} & r_{12} \\
r_{21} & r_{22}
\end{array}\right), \quad R^{\sharp}=\left(\begin{array}{cc}
r_{22}^{*} & r_{12}^{*} \\
r_{21}^{*} & r_{11}^{*}
\end{array}\right)
$$

yields

$$
r_{12}=0, \quad \frac{\alpha_{1}}{\alpha_{0}}=\frac{r_{11}}{r_{22}}=\frac{r_{11}^{*}}{r_{22}^{*}}
$$

Hence, we can set

$$
r_{11}=\left|r_{11}\right| e^{i \gamma}, \quad r_{22}=\left|r_{22}\right| e^{i \gamma}, \quad r_{21}=\left|r_{21}\right| e^{i(\gamma+\varepsilon)} .
$$

Using this and (19) in

$$
R R^{\sharp}=K_{0}=\left(\begin{array}{cc}
1 & 0 \\
-\alpha_{0} & 1
\end{array}\right), \quad R^{\sharp} R=K_{1}=\left(\begin{array}{cc}
1 & 0 \\
-\alpha_{1} & 1
\end{array}\right)
$$

we find

$$
R=e^{i \gamma}\left(\begin{array}{cc}
\sqrt{\frac{\alpha_{1}}{\alpha_{0}}} & 0 \\
-\frac{1}{2} \sqrt{\alpha_{0} \alpha_{1}}(1+i \tan \varepsilon) & \sqrt{\frac{\alpha_{0}}{\alpha_{1}}}
\end{array}\right),
$$

where the phases $\gamma$ and $\varepsilon$ are still undefined.

As next step we analyze Eqs. (14) and (17). It is easily seen that defining the matrices

$$
U:=R\left[Q^{\sharp}-\left(R^{\sharp}\right)^{\prime}\right], \quad B:=R^{\sharp} Q
$$

these equations are equivalent to the $J$-symmetry relations

$$
U=U^{\sharp}, \quad B=B^{\sharp} .
$$

Due to the different symmetry content of $B$ and $Q$ it is natural to consider $B$ as primary structural element of the intertwining construction, and $Q$ as a secondary one. So, we perform our subsequent investigation in terms of $B$ and $R$. Explicitly, the $J$-symmetry is realized by the matrix structure

$$
B=\left(\begin{array}{cc}
b_{1}+i b_{4} & b_{2} \\
b_{3} & b_{1}-i b_{4}
\end{array}\right), \quad \operatorname{Im} b_{k}=0, \quad k=1, \ldots, 4 .
$$

Furthermore, we exclude $Q$ from (21) to obtain

$$
U=R B R^{-1}-R\left(R^{\sharp}\right)^{\prime} .
$$


Introducing the notation $N:=R^{-1} R^{\prime}$ and substituting (23) into the symmetry relation $U=U^{\sharp}$ yields the additional constraint

$$
\left[B, K_{1}^{-1}\right]=N^{\sharp}-N \text {. }
$$

From eq. (20) we find

$$
\begin{aligned}
N & =i \gamma^{\prime} I+\left(\begin{array}{cc}
-q & 0 \\
f & q
\end{array}\right) \\
q & =\frac{1}{2}\left(\frac{\alpha_{0}^{\prime}}{\alpha_{0}}-\frac{\alpha_{1}^{\prime}}{\alpha_{1}}\right) \\
f & =-\frac{\alpha_{1}}{2}\left[\frac{\alpha_{0}^{\prime}}{\alpha_{0}}(1+i \tan \varepsilon)+i \frac{\varepsilon^{\prime}}{\cos ^{2} \varepsilon}\right]
\end{aligned}
$$

so that (24) transforms to

$$
\alpha_{1}\left(\begin{array}{cc}
b_{2} & 0 \\
-2 i b_{4} & -b_{2}
\end{array}\right)=-2 i \gamma^{\prime} I+\left(\begin{array}{cc}
2 q & 0 \\
f^{*}-f & -2 q
\end{array}\right)
$$

Finally, we arrive at the following restrictions on the phase $\gamma$ and the components $b_{2}$ and $b_{4}$ of the matrix $B$ :

$$
\gamma^{\prime}=0, \quad b_{2}=\frac{2 q}{\alpha_{1}}, \quad b_{4}=\frac{\operatorname{Im} f}{\alpha_{1}}=-\frac{1}{2}\left(\frac{\alpha_{0}^{\prime}}{\alpha_{0}} \tan \varepsilon+\frac{\varepsilon^{\prime}}{\cos ^{2} \varepsilon}\right) .
$$

Summarizing the implications of the first four consistency conditions we see that they are free of intrinsic contradictions. From the initially eight arbitrary complex-valued functions contained in the matrices $R$ and $Q$ only the three real-valued functions $\left(b_{1}, b_{3}, \varepsilon\right)$ are still undefined. Together with the helical turbulence functions $\left(\alpha_{0}, \alpha_{1}\right)$ and the constants $\left(\gamma, E, l_{0}, l_{1}\right) \in \mathbb{R}^{2} \times \mathbb{Z}_{+}^{2}$ we expect them to be highly fine-tuned by the remaining two consistency conditions (15) and (18).

Let us study these conditions now. Making use of the definitions of $U$ and $B$ in (21), their implications

$$
\begin{aligned}
Q^{\sharp}-\left(R^{\sharp}\right)^{\prime} & =R^{-1} U, \\
\left(Q^{\sharp}\right)^{\prime}-\left(R^{\sharp}\right)^{\prime \prime} & =-R^{-1} R^{\prime} R^{-1} U+R^{-1} U^{\prime}, \\
Q & =\left(R^{\sharp}\right)^{-1} B
\end{aligned}
$$

and setting at the end $R R^{\sharp}=K_{0}, R^{\sharp} R=K_{1}$ according to eqs. (13), (16), we find that (15) and (18) transform to the matrix Riccati equations (MREs)

$$
\begin{aligned}
U^{\prime} & =M_{0}-U K_{0}^{-1} U, \\
B^{\prime} & =-M_{1}+B K_{1}^{-1} B .
\end{aligned}
$$

Similar to the linearization of the scalar Riccati equations mentioned in (3) - (6) of the Introduction, the MREs (30), (31) can be linearized by an Ansatz 23, 24]

$$
\begin{aligned}
& U=V W^{-1}, \quad V, W \in \mathbb{C}^{2 \times 2}, \quad \operatorname{det}(W) \neq 0, \\
& B=X Y^{-1}, \quad X, Y \in \mathbb{C}^{2 \times 2}, \quad \operatorname{det}(Y) \neq 0 .
\end{aligned}
$$

As result we arrive at the equation systems

$$
\left(\begin{array}{c}
V^{\prime} \\
W^{\prime}
\end{array}\right)=\left(\begin{array}{cc}
0 & M_{0} \\
K_{0}^{-1} & 0
\end{array}\right)\left(\begin{array}{c}
V \\
W
\end{array}\right), \quad\left(\begin{array}{c}
X^{\prime} \\
Y^{\prime}
\end{array}\right)=-\left(\begin{array}{cc}
0 & M_{1} \\
K_{1}^{-1} & 0
\end{array}\right)\left(\begin{array}{c}
X \\
Y
\end{array}\right) .
$$

The $4 \times 2$ matrices $\left(\begin{array}{c}V \\ W\end{array}\right),\left(\begin{array}{c}X \\ Y\end{array}\right) \in \mathbb{C}^{4 \times 2}$ are defined up to $G L(2, \mathbb{C}) \times G L(2, \mathbb{C})$-transformations

$$
\left(\begin{array}{c}
\tilde{V} \\
\tilde{W}
\end{array}\right)=\left(\begin{array}{c}
V G_{0} \\
W G_{0}
\end{array}\right), \quad\left(\begin{array}{c}
\tilde{X} \\
\tilde{Y}
\end{array}\right)=\left(\begin{array}{c}
X G_{1} \\
Y G_{1}
\end{array}\right), \quad G_{0}, G_{1} \in G L(2, \mathbb{C})
$$


and can be interpreted as homogeneous coordinates of two points on a complex Grassmann manifold $G_{2}\left(\mathbb{C}^{4}\right)$ which consists of 2 -dimensional complex subspaces in $\mathbb{C}^{4}$ (see, e.g. 23, 24]). The matrices $U=V W^{-1}$ and $B=X Y^{-1}$ are the corresponding affine coordinates of these points.

Differentiating (34) and substituting $V=K_{0} W^{\prime}, X=-K_{1} Y^{\prime}$ it is easily seen that the equation systems (34) are equivalent to the second-order matrix differential equations

$$
\begin{aligned}
\left(\partial_{r} K_{0} \partial_{r}-M_{0}\right) W & =0 \\
\left(\partial_{r} K_{1} \partial_{r}-M_{1}\right) Y & =0 .
\end{aligned}
$$

This implies that the matrices $\tilde{W}=r^{-1} W, \tilde{Y}=r^{-1} Y$ should be formal (non-normalized) solutions of the eigenvalue equations for the dynamo operator matrices $\hat{H}_{l_{0}}\left[\alpha_{0}\right], \hat{H}_{l_{1}}\left[\alpha_{1}\right]$, respectively

$$
\hat{H}_{l_{0}}\left[\alpha_{0}\right] \tilde{W}=E \tilde{W}, \quad \hat{H}_{l_{1}}\left[\alpha_{1}\right] \tilde{Y}=E \tilde{Y} .
$$

A comparison with the simple QM model from the Introduction shows that the intertwining operator matrix $\hat{A}$ should be expressible in terms of $W$ or $Y$, and that $W$ and $Y$ should be connected by a product invariant like (7). With the help of (28), (29) and (34) we find

$$
\hat{A}=R\left(i p-Y^{\prime} Y^{-1}\right)=\left(i p+K_{0} W^{\prime} W^{-1} K_{0}^{-1}\right) R .
$$

In order to obtain the product invariant which connects $W$ and $Y$, we use a slightly modified version of (23)

$$
U=R B^{\sharp} R^{-1}-R\left(R^{\sharp}\right)^{\prime}
$$

and substitute from (32) - (34)

$$
U=R R^{\sharp} W^{\prime} W^{-1}, \quad B^{\sharp}=-\left(Y^{\sharp}\right)^{-1}\left(Y^{\sharp}\right)^{\prime} R^{\sharp} R
$$

so that

$$
W^{\prime} W^{-1}=-\left(R^{\sharp}\right)^{-1}\left(Y^{\sharp}\right)^{-1}\left(Y^{\sharp}\right)^{\prime} R^{\sharp}-\left(R^{\sharp}\right)^{-1}\left(R^{\sharp}\right)^{\prime} .
$$

This equation is of the type $g=g_{1} n,\left(\partial_{r} g\right) g^{-1}=\left(\partial_{r} g_{1}\right) g_{1}^{-1}+g_{1}\left(\partial_{r} n\right) n^{-1} g_{1}^{-1}$. Hence, integration of (36) yields the product invariant

$$
Y^{\sharp} R^{\sharp} W=C, \quad \operatorname{det}(C) \neq 0,
$$

with $C$ a constant non-singular matrix.

So far, we have obtained a $1: 1$ generalization of the intertwining technique from the simple QM example described in the Introduction to our $J$-symmetric dynamo operator model. It remains to test whether the MREs of this model are consistent. This will be the subject of the next section.

\section{No-go theorem}

In order to test the pair of MREs (30), (31) for consistency, we make use of (23), (24) as well as the relation

$$
N+K_{1}^{-1} N^{\sharp} K_{1}=K_{1}^{-1} K_{1}^{\prime}=K_{1}^{\prime}
$$

and transform the MRE for $U$ [eq. [30] into an equivalent MRE for $B$. As result, we arrive at the following pair of MREs

$$
\begin{aligned}
& B^{\prime}=R^{-1} M_{0} R-K_{1}^{-1} B B+B K_{1}^{\prime}+\left[N N^{\sharp}+\left(N^{\sharp}\right)^{\prime}\right] K_{1}, \\
& B^{\prime}=-M_{1}+B K_{1}^{-1} B
\end{aligned}
$$

which should be satisfied simultaneously. The corresponding consistency test will be performed in two steps:

1. From the limiting behavior at $r \rightarrow 0$ we will derive a relation between $l_{0}$ and $l_{1}$.

2. We will extract from eqs. (37), (38) a system of non-linear ODEs for the helical turbulence functions $\alpha_{0}, \alpha_{1}$ and for the components $b_{1}, \ldots, b_{4}$ of the matrix $B$. By mutual substitutions of these ODEs we will find an inconsistency which can be interpreted as a no-go theorem. 


\section{4, 1 Limiting behavior at $r \rightarrow 0$}

From the assumed non-singular behavior of the helical turbulence functions at $r \rightarrow 0$ follows that they can be approximated as

$$
\alpha_{0,1}(r \rightarrow 0) \approx c_{0,1}+a_{0,1} r+\mathcal{O}\left(r^{2}\right), \quad c_{0,1} \neq 0 .
$$

Substituting this approximation in a slightly rewritten version of the defining equation (35) for the matrix $Y$

$$
\left[I \partial_{r}^{2}-\alpha_{1}^{\prime} \sigma_{-} \partial_{r}-\frac{l_{1}\left(l_{1}+1\right)}{r^{2}} I-\left(\begin{array}{cc}
E & -\alpha_{1} \\
\alpha_{1} & E-\alpha_{1}^{2}
\end{array}\right)\right] Y=0
$$

we obtain the estimate

$$
Y(r \rightarrow 0) \approx r^{-l_{1}}\left(I+\frac{a_{1}}{2} \sigma_{-} r+\mathcal{O}\left(r^{2}\right)\right)\left(r^{2 l_{1}+1} C_{+}+C_{-}\right),
$$

where $C_{+}, C_{-}$are arbitrary non-singular constant matrices $\operatorname{det}\left(C_{ \pm}\right) \neq 0$. Correspondingly, it holds

$$
\begin{aligned}
Z:=Y^{\prime} Y^{-1} & \approx-l_{1} r^{-1} I+\frac{a_{1}}{2} \sigma_{-}+\mathcal{O}(r), \\
B=-K_{1} Y^{\prime} Y^{-1} & \approx l_{1} r^{-1} I-\left[c_{1} l_{1} r^{-1}+\left(l_{1}+1 / 2\right) a_{1}\right] \sigma_{-}+\mathcal{O}(r) .
\end{aligned}
$$

Comparison of (41) with (22) shows that the components $b_{2}$ and $b_{4}$ of the matrix $B$ vanish at least as

$$
b_{2}, b_{4} \approx \mathcal{O}(r) \quad \text { for } r \rightarrow 0 \text {. }
$$

Furthermore, we find with the help of eqs. (25), (26) and (27) that $q \approx \mathcal{O}(r)$ and, hence, $a_{0} / c_{0}=a_{1} / c_{1}$, as well as $q^{\prime}, f, f^{\prime} \approx \mathcal{O}(1)$ what implies $N, N^{\sharp},\left(N^{\sharp}\right)^{\prime} \approx \mathcal{O}(1)$.

We are now well prepared to perform a partial consistency test of (37) and (38) by comparing the singular terms of these equations in the vicinity of the origin $r=0$. From the MREs (37) and (38) we find

$$
\begin{aligned}
-K_{1}^{-1} K_{1}^{\prime} Z-Z^{\prime} & =\frac{l_{0}\left(l_{0}+1\right)}{r^{2}} I-K_{1}^{-1} Z K_{1} Z-Z K_{1}^{\prime}+\mathcal{O}(1), \\
-K_{1}^{-1} K_{1}^{\prime} Z-Z^{\prime} & =-\frac{l_{1}\left(l_{1}+1\right)}{r^{2}} I+Z Z+\mathcal{O}(1),
\end{aligned}
$$

respectively. Substituting $Z$ from (40) and equating the coefficients of the $r^{-2}, r^{-1}$-terms we obtain from eq. (42)

$$
l_{1}=l_{0}+1, \quad a_{1}=0
$$

and, hence, also $a_{0}=0$. Eq. (43) is automatically satisfied, because $Y$ is defined by the corresponding linearized equation (39). The incremental relation $l_{1}=l_{0}+1$ is well known from ladder operator constructions for spherically symmetric Hamiltonians in QM [5]. This is not surprising, because this ladder operator construction can be recovered from the intertwining construction (10) for the $\alpha^{2}$-dynamo operator matrices by the two-step transition: $1 . \alpha_{0}=\alpha_{1}=\alpha, 2 . \alpha \rightarrow 0$.

4.2 Systems of coupled non-linear ODEs and their inconsistency

The system of eight coupled non-linear ODEs for the components $b_{1}, \ldots, b_{4}$ of the matrix $B$ is easily obtained from the MREs (37), (38), e.g. with the help of the matrix multiplication package of MATHEMATICA ${ }^{\odot}$. For our analysis it is sufficient to consider only the simplest four equations of this system, i.e. the $\sigma_{+}$and $I$ projections of (37) and (38):

$$
\begin{aligned}
b_{2}^{\prime} & =2 b_{1} b_{2}+\alpha_{1}\left(1+b_{2}^{2}\right) \\
& =-2 b_{1} b_{2}-\frac{\alpha_{0}^{2}}{\alpha_{1}} \\
b_{1}^{\prime} & =b_{1}^{2}+b_{2} b_{3}-b_{4}^{2}-E-\frac{l_{1}\left(l_{1}+1\right)}{r^{2}}+\alpha_{1} b_{1} b_{2} \\
& =-b_{1}^{2}-b_{2} b_{3}+b_{4}^{2}+E+\frac{l_{0}\left(l_{0}+1\right)}{r^{2}}-\alpha_{1}^{\prime} b_{2}+\frac{\alpha_{0}^{2}}{2}+q^{\prime}-q^{2} .
\end{aligned}
$$

Equating the right-hand-sides of (44), (45) and using $b_{2}=2 q / \alpha_{1}$ from (27) we are able to express $b_{1}$ as

$$
b_{1}=-\frac{4 q^{2}+\alpha_{0}^{2}+\alpha_{1}^{2}}{8 q} .
$$


Taking into account that $q=\partial_{r} \ln \left(\alpha_{0} / \alpha_{1}\right) / 2$ according to (25) and that the helical turbulence functions $\alpha_{0}$ and $\alpha_{1}$ do not depend on $l_{0}$ or $l_{1}$ we conclude from equation (48) that $b_{1}$ should not depend on $l_{0}$ or $l_{1}$ too. On the other hand, addition of (46) and (47) together with the relation $l_{0}=l_{1}-1$ gives

$$
2 b_{1}^{\prime}=-\frac{2 l_{1}}{r^{2}}+2 q\left(b_{1}-\frac{\alpha_{1}^{\prime}}{\alpha_{1}}\right)+\frac{\alpha_{0}^{2}}{2}+q^{\prime}-q^{2}
$$

what by integration leads to a function $b_{1}$ which depends on $l_{1}$. I.e. the term depending on $l_{1}$ cannot be compensated by a combination of $l_{1}$-independent terms. This is an obvious contradiction to (48) and we have to conclude that the consistency conditions (13) - (18) cannot be fulfilled simultaneously. This means that we are lead to the

No-go theorem:

The structure of the MHD $\alpha^{2}$-dynamo operator matrix is incompatible with an operator intertwining technique which is based on first-order differential intertwining operators.

A similar situation occurs also for three-dimensional spherically symmetric models in QM [5]. There the $l$-dependent centrifugal term sets so strong restrictions on the form of the allowed potential that an intertwining construction built on first-order differential intertwining operators is only possible for the following three cases: the constant potential $V(r)=$ const, the Coulomb potential $V(r) \propto 1 / r$, and the potential of the threedimensional isotropic harmonic oscillator with $V(r) \propto r^{2}$. Richer classes of allowed potentials are only found for models in their $s$ states, when $l=0$. Such states are a priori excluded for the $\alpha^{2}$-dynamo operator matrix due to its construction [see Eq. A5 ]

\section{Concluding remarks}

In the present paper, we have tested the MHD $\alpha^{2}$-dynamo operator matrix for its compatibility with the simplest variant of an intertwining construction based on first-order differential intertwining operators. The operators have been chosen in accordance with the fundamental $J$-symmetry (pseudo-Hermiticity) of the operator matrix and lead to a set of six matrix equations as consistency conditions. With the help of a stepby-step reduction of the complexity we have extracted their basic structural elements and have shown that they contain an intrinsic inconsistency. So, we have to conclude that the structure of the $\alpha^{2}$-dynamo operator matrix is not compatible with the considered first-order differential intertwining Ansatz. This fact is the subject of the formulated no-go theorem.

It remains to test whether intertwining constructions can be built from second-order or higher-order differential intertwining operators. Energy shift operators based on second-order differential expressions are known for harmonic oscillators with time-dependent frequencies and additional $1 / r^{2}-$ term [25] as well as for the spherically symmetric oscillator and the Coulomb potential [5]. A generalization of the technique to the MHD $\alpha^{2}-$ dynamo operator matrix seems realistic.

Another approach for a clarification of the considered isospectrality problem could consist in a generalization of the Gelfand-Levitan technique for vector-valued Sturm-Liouville problems [26. Concerning its general structure, the $\alpha^{2}$-dynamo operator matrix $\hat{H}_{l}[\alpha]$ is a singular non-self-adjoint matrix Sturm-Liouville operator which by a unitary transformation can be recast into the standard form

$$
-\partial_{r} P_{2}(r) \partial_{r}+P_{0}(r) \text {. }
$$

In 1998, Jodeit and Levitan [26] analyzed the isospectrality problem for matrix Sturm-Liouville operators with $P_{2}(r)=I$ and $P_{0}(r)$ a symmetric matrix. They showed that if two vector-valued Sturm-Liouville problems are isospectral then the eigenfunctions of one problem can be constructed from the eigenfunctions of the other problem with the help of a matrix Gelfand-Levitan transformation. So, a generalization of this technique to Sturm-Liouville problems with non-symmetric $P_{0}(r)$ and $P_{2}(r) \neq I_{2}$ would naturally cover the isospectrality problem for the MHD $\alpha^{2}$-dynamo operator matrix.

\section{Acknowledgements}

We would like to thank G. Gerbeth for numerous discussions and C. Tretter for useful comments. This project was supported by the German Research Foundation, DFG, under grant GE-682/12-1.

\section{A Derivation of the $\alpha^{2}$-dynamo operator matrix from the mean- field induction equation}

For completeness we sketch here the main steps of the derivation of the $2 \times 2$ operator matrix $\hat{H}_{l}[\alpha]$ for a model with helical turbulence function $\alpha(r)$. The outline follows the technique for models with $\alpha=$ const as presented 
in 1 .

The spherical MHD mean-field $\alpha^{2}$-dynamo in its kinematic regime is described by the induction equation for the magnetic field

$$
\partial_{t} \mathbf{B}=\boldsymbol{\nabla} \times(\alpha \mathbf{B})+\nu_{m} \Delta \mathbf{B}
$$

supplemented by the condition $\boldsymbol{\nabla} \cdot \mathbf{B}=0$. The magnetic diffusivity $\nu_{m}$ is assumed to be constant and the helical turbulence function $\alpha$ to depend only on the distance from the origin $\alpha=\alpha(r)$. Decomposition into toroidal and poloidal components $\mathbf{B}=\mathbf{B}_{\mathbf{t}}+\mathbf{B}_{\mathbf{p}}$ and setting $\mathbf{B}_{\mathbf{p}}=\boldsymbol{\nabla} \times \mathbf{A}_{\mathbf{t}}$ allows for a decomposition of the induction equation (A1)

$$
\begin{aligned}
\partial_{t} \mathbf{B}_{\mathbf{t}} & =\boldsymbol{\nabla} \times\left(\alpha \boldsymbol{\nabla} \times \mathbf{A}_{\mathbf{t}}\right)-\nu_{m} \boldsymbol{\nabla} \times \boldsymbol{\nabla} \times \mathbf{B}_{\mathbf{t}} \\
\partial_{t} \mathbf{A}_{\mathbf{t}} & =\alpha \mathbf{B}_{\mathbf{t}}-\nu_{m} \boldsymbol{\nabla} \times \boldsymbol{\nabla} \times \mathbf{A}_{\mathbf{t}} .
\end{aligned}
$$

Furthermore, the fields $\mathbf{B}_{\mathbf{t}}$ and $\mathbf{A}_{\mathbf{t}}$ can be represented as

$$
\mathbf{A}_{\mathbf{t}}=-\mathbf{r} \times \nabla F_{1}, \quad \mathbf{B}_{\mathbf{t}}=-\mathbf{r} \times \nabla F_{2},
$$

where $F_{1}$ and $F_{2}$ are single-valued scalar functions which are normalized on the unit sphere $S^{2}$ by the condition

$$
\int_{S^{2}} F_{1,2} d \omega=0
$$

With the help of the relations

$$
\begin{aligned}
\Delta\left(\mathbf{r} \times \nabla F_{1}\right) & =\mathbf{r} \times \nabla \Delta F_{1} \\
\nabla \times\left[\alpha \nabla \times\left(-\mathbf{r} \times \nabla F_{1}\right)\right] & =\mathbf{r} \times \nabla\left[\frac{1}{r}\left(\partial_{r} \alpha\right)\left(\partial_{r} r F_{1}\right)+\alpha \Delta F_{1}\right], \\
\alpha \mathbf{r} \times \nabla F_{2} & =\mathbf{r} \times \nabla\left(\alpha F_{2}\right)
\end{aligned}
$$

equations (A2) and (A3) can be rewritten as

$$
\begin{aligned}
\mathbf{r} \times \boldsymbol{\nabla}\left[\nu_{m} \Delta F_{1}+\alpha F_{2}-\partial_{t} F_{1}\right] & =0, \\
\mathbf{r} \times \nabla\left[\nu_{m} \Delta F_{2}-\frac{1}{r}\left(\partial_{r} \alpha\right)\left(\partial_{r} r F_{1}\right)-\alpha \Delta F_{1}-\partial_{t} F_{2}\right] & =0 .
\end{aligned}
$$

It follows that the expressions in the square brackets are functions of $r$ and $t$ alone which must vanish due to the normalization condition (A4) and its implication $\int_{S^{2}} \Delta F_{1,2} d \omega=0$. By re-scaling of $r$ and $t$ one sets the magnetic diffusivity to unity $\nu_{m}=1$ and the boundary conditions at $r=1$.

With the help of a series expansion in spherical harmonics

$$
F_{1,2}=\sum_{l, m, n} e^{\lambda_{l, n} t} F_{1,2}^{(l, m, n)}(r) Y_{l}^{m}(\theta, \phi) \in L^{2}\left(\Omega, r^{2} d r\right) \otimes L^{2}\left(S^{2}, d \omega\right), \quad \Omega=[0,1]
$$

one obtains the eigenvalue problem

$$
\begin{aligned}
\Delta_{l} F_{1}^{(l, m, n)}+\alpha F_{2}^{(l, m, n)} & =\lambda_{l, n} F_{1}^{(l, m, n)} \\
\Delta_{l} F_{2}^{(l, m, n)}-\frac{1}{r}\left(\partial_{r} \alpha\right)\left(\partial_{r} r F_{1}^{(l, m, n)}\right)-\alpha \Delta_{l} F_{1}^{(l, m, n)} & =\lambda_{l, n} F_{2}^{(l, m, n)} .
\end{aligned}
$$

Here we used the notation $\Delta_{l}=\frac{1}{r^{2}} \partial_{r} r^{2} \partial_{r}-\frac{l(l+1)}{r^{2}}$ and the fact that due to the symmetry of the dynamo configuration [1] the eigenvalues $\lambda_{l, n}$ depend only on $l$ and $n$. We note that the normalization condition (A4) implies

$$
F_{1,2}^{(l=0, m, n)}=0 .
$$

Finally, the substitutions $p=-i\left(\partial_{r}+1 / r\right), \quad \psi_{1,2}=F_{1,2}^{(l, m, n)} \in L^{2}\left(\Omega, r^{2} d r\right)$ lead to the eigenvalue problem for the $\alpha^{2}$-dynamo operator matrix $\hat{H}_{l}[\alpha]$ as it is given in eq. (8) of the Introduction. 


\section{References}

[1] H. K. Moffatt, Magnetic field generation in electrically conducting fluids (Cambridge University Press, Cambridge, 1978); F. Krause and K.-H. Rädler, Mean-field magnetohydrodynamics and dynamo theory (Akademie-Verlag, Berlin and Pergamon Press, Oxford, 1980); Ya. B. Zeldovich, A. A. Ruzmaikin, and D. D. Sokoloff, Magnetic fields in astrophysics (Gordon \& Breach Science Publishers, New York, 1983).

[2] A. Gailitis et al., Phys. Rev. Lett. 84, 4365 (2000); Phys. Rev. Lett. 86, 3024 (2001), physics/0010047 U. Müller and R. Stieglitz, Phys. Fluids 13, 561 (2001); A. Gailitis et al., Rev. Mod. Phys. 74, 973 (2002).

[3] F. Stefani and G. Gerbeth, Astron. Nachr. 321, 235 (2000), astro-ph/0010090

[4] F. Stefani and G. Gerbeth, Phys. Earth Planet. Inter. 128, 109 (2001); Phys. Rev. E 67, 027302 (2003), astro-ph/0210412

[5] O. L. De Lange and R. E. Raab, Operator methods in quantum mechanics (Clarendon Press, Oxford, 1991).

[6] V. B. Matveev and M. A. Salle, Darboux transformations and solitons (Springer, Berlin, 1991).

[7] D. L. Pursey, Phys. Rev. D 33, 2267 (1986); A. Anderson, Phys. Rev. A 43, 4602 (1991).

[8] A. Gonzalez-Lopez and N. Kamran, J. Geom. Phys. 26, 202 (1998), hep-th/9612100

[9] E. Witten, Nucl. Phys. B 202, 253 (1982); L. E. Gendenstein and I. V. Krive, Sov. Phys. Usp. 28, 645 (1985); F. Cooper, A. Khare, and U. Sukhatme, Phys. Rep. 251, 267 (1995).

[10] A. Mostafazadeh, J. Math. Phys. 43, 205 (2002), math-ph/0107001.

[11] A. Mostafazadeh, Nucl. Phys. B 640, 419 (2002), math-ph/0203041.

[12] F. Gesztesy, J. Funct. Anal. 117, 401 (1993); F. Gesztesy and G. Teschl, Proc. Am. Math. Soc. 124, 1831 (1996); F. Gesztesy and G. Teschl, J. Differ. Equations 128, 252 (1996).

[13] M. Proctor, Astron. Nachr. 298, 19 (1977).

[14] T. Ya. Azizov and I. S. Iokhvidov, Linear operators in spaces with an indefinite metric (Wiley-Interscience, New York, 1989).

[15] H. Langer and C. Tretter, in H. Bart (ed.) et al., Operator theory and analysis, Operator Theory: Adv. Appl. 122, 331 (2001).

[16] A. Mostafazadeh, J. Math. Phys. 44, 974 (2003), math-ph/0209018 C. M. Bender, D. C. Brody, and H. F. Jones, Must a hamiltonian be hermitian?, hep-th/0303005.

[17] A. Mostafazadeh, J. Math. Phys. 43, 2814 (2002), math-ph/0110016 J. Math. Phys. 43, 3944 (2002), math-ph/0203005 Mod. Phys. Lett. A 17, 1973 (2002), math-ph/0204013 J. Math. Phys. 43, 6343 (2002), math-ph/0207009.

[18] S. Albeverio, F. Gesztesy, R.H. Krohn, and H. Holden, Solvable models in quantum mechanics (Springer, New York, 1988).

[19] V. I. Gorbachuk and M. L. Gorbachuk, Boundary value problems for operator differential equations (Kluwer Academic Publishers, Dortrecht, 1991); E. M. Russakovskii, Linear Algebra Appl. 212/213, 437 (1994).

[20] A. Dijksma and H. Langer, Operator theory and ordinary differential operators, in A. Böttcher (ed.) et al., Lectures on operator theory and its applications, Providence, RI: Am. Math. Soc., Fields Institute Monographs, 3, 75 (1996).

[21] A. S. Markus, Introduction to the spectral theory of polynomial operator pencils (Translations of Mathematical Monographs, 71. Providence, RI: Am. Math. Soc., 1988); Yu. S. Abramov, Variational methods in the theory of operator pencils - Spectral optimization, (in Russian, Izd. Leningrad. Univ., Leningrad, 1983).

[22] I. C. Gohberg and M. G. Krein, Introduction to the theory of linear nonselfadjoint operators (Translations of Mathematical Monographs, 18. Providence, RI: Am. Math. Soc., 1969); T. Kato, Perturbation theory of linear operators (Springer, Berlin, 1980); H. Baumgärtel, Analytic perturbation theory for matrices and operators (Akademie-Verlag, Berlin, 1984, and Operator Theory: Adv. Appl 15, Birkhäuser Verlag, Basel, 1985). 
[23] M. I. Zelikin, Control theory and optimization I. Homogeneous spaces and the Riccati equation in the calculus of variations (Springer, Berlin, 2000).

[24] R. L. Anderson, J. Harnad, and P. Winternitz, J. Math. Phys. 24, 1062 (1983); S. Shnider and P. Winternitz, J. Math. Phys. 25, 3155 (1984); S. Lafortune and P. Winternitz, J. Math. Phys. 37, 1539 (1996).

[25] P. Camiz et al., J. Math. Phys. 12, 2040 (1971).

[26] M. Jodeit and B. M. Levitan, Lett. Math. Phys. 43, 117 (1998). 